\title{
Norois
}

Environnement, aménagement, société

$210 \mid 2009 / 1$

L'agriculture et les filières agro-alimentaires en France et en Argentine

\section{La filière des agrumes doux dans la province d'Entre Ríos, Argentine}

The sweet citrus fruits agro-industrial complex in the province of Entre Ríos La frutícultura en la Argentina desde los años 90. El caso del complejo

agroindustrial de cítricos dulces en la provincia de Entre Ríos. Argentina

\section{Nidia Tadeo}

\section{(2) OpenEdition}

Journals

Édition électronique

URL : https://journals.openedition.org/norois/2790

DOI : $10.4000 /$ norois. 2790

ISBN : 978-2-7535-1558-1

ISSN : $1760-8546$

\section{Éditeur}

Presses universitaires de Rennes

Édition imprimée

Date de publication : 1 mars 2009

Pagination : 69-80

ISBN : 978-2-7535-0844-6

ISSN : 0029-182X

Référence électronique

Nidia Tadeo, «La filière des agrumes doux dans la province d'Entre Ríos, Argentine », Norois [En ligne], 210 | 2009/1, mis en ligne le 01 mars 2011, consulté le 13 janvier 2022. URL : http:// journals.openedition.org/norois/2790; DOI : https://doi.org/10.4000/norois.2790

Ce document a été généré automatiquement le 13 janvier 2022.

(c) Tous droits réservés 


\title{
La filière des agrumes doux dans la province d'Entre Ríos, Argentine
}

\author{
The sweet citrus fruits agro-industrial complex in the province of Entre Ríos \\ La frutícultura en la Argentina desde los años 90. El caso del complejo \\ agroindustrial de cítricos dulces en la provincia de Entre Ríos. Argentina
}

Nidia Tadeo

\section{NOTE DE L'ÉDITEUR}

Cet article a été reçu le 22 juin 2008 et définitivement accepté le 15 janvier 2009.

1 Le processus de restructuration économique qui a pris place en Argentine dans les années 1990, a été fondé sur une ouverture externe plus importante, la privatisation d'entreprises publiques, l'éloignement de l'État en tant qu'agent de surveillance et dispensateur de ressources, et le régime monétaire de convertibilité1. Ce contexte a accéléré la pénétration et la concentration du capital international dans les territoires ruraux du pays. L'expansion des filières agro-industrielles est une expression de la présence du capital agro-industriel, un phénomène dont la manifestation n'a pas été homogène et qui a adopté diverses modalités d'insertion et de rapports productifs. Les possibilités d'accéder aux marchés globaux, où le capital international planifie ses affaires à échelle mondiale, entraînent une nouvelle adéquation constante de la structure des exploitations agricoles et des entreprises agro-industrielles afin de répondre aux exigences d'une concurrence croissante, de standards de qualité et des règles de consommation changeantes, d'une production régulière tant en quantité qu'en qualité. Parallèlement, la rentabilité augmente et les coûts de production creusent des inégalités parmi les acteurs impliqués dans les agro-industries. Ce processus qui persiste dans le contexte économique de la posconvertibilité 2 depuis janvier 2002, a eu une incidence inégale sur les chaînes agro-industrielles ; on peut remarquer 
des progrès, des reculs, des alliances, des conflits, des tensions, de nouvelles formes d'intégration et d'articulation.

2 Au cours de ces années 1990, la demande de fruits frais a progressé sur le marché mondial. Les grandes entreprises agro-industrielles argentines ont alors essayé de faire face à la stagnation économique, aux difficultés financières et à la diminution de gains par la chute de la consommation nationale, en intensifiant l'exportation de produits frais destinés au marché de contre-saison. Au fur et à mesure, elles ont réadapté leur processus productif ainsi que leur gestion interne (la structure administrative, l'organisation et les conditions du travail) et l'organisation territoriale de leurs activités pour disposer des volumes d'exportation plus importants et certifier la qualité de leurs produits par l'application des normes de qualité.

3 Cet article observe l'importance atteinte par la production et la commercialisation de fruits frais argentins destinés au marché extérieur de contre-saison depuis 1990, comme une introduction à l'analyse de la filière agro-industrielle agrumicole d'Entre Ríos et sa restructuration productive depuis la dernière décennie. Les approches empirique et analytique de cette étude ont favorisé l'utilisation d'informations qualitatives recueillies en 2005 et 2006 (des enquêtes directes aux entreprises d'emballage des agrumes de table et des entretiens aux producteurs agrumicoles), accompagnées de données statistiques.

\section{La filière des agrumes doux de l'Entre Ríos dans la fruiticulture argentine}

\section{La production de fruits en Argentine}

4 Depuis une quinzaine d'années, la fruiticulture est une des productions clés de l'agriculture argentine, élément dynamique de l'exportation des produits de contresaison vers les principaux marchés de consommation (fig. 1 et tableau 1). L'Argentine a une position stratégique pour la vente de fruits frais vers l'hémisphère nord et occupe la sixième place au rang des filières agro-exportatrices nationales (Institut National de Statistiques et Recensements-INDEC, 2006). Les entreprises qui ont intégré le maillon de la chaîne d'exportation, se sont modernisées par l'utilisation de technologie de pointe, des économies d'échelle, la décentralisation des processus productifs, ainsi comme par le contrôle des voies de commercialisation et de distribution. Si on compare cette activité avec celle des produits traditionnels de l'Argentine (viande et grains), on peut observer que son importance est moindre pour le PBI agricole et la génération de devises. Pourtant, la valeur des ventes sur le marché extérieur a augmenté de 8,5\% au cours des années 1990 (Reca et Parellada, 2001). Le Recensement National Agricole de 2002 indique que $4 \%$ de la superficie totale cultivée du pays est dédiée à la fruiticulture (soit 544217 ha en Argentine). Entre 2000 et 2006, environ 600000 ha de fruitiers ont été plantés dans différentes régions et la production moyenne annuelle a été de 7700 000 tonnes (Secrétariat à l'Agriculture, l'Élevage, la Pêche et l'Alimentation (SAGPyA, 2007). 
Figure 1. Les zones de production des fruits d'exportation Map of the mains regions producing fruits for export

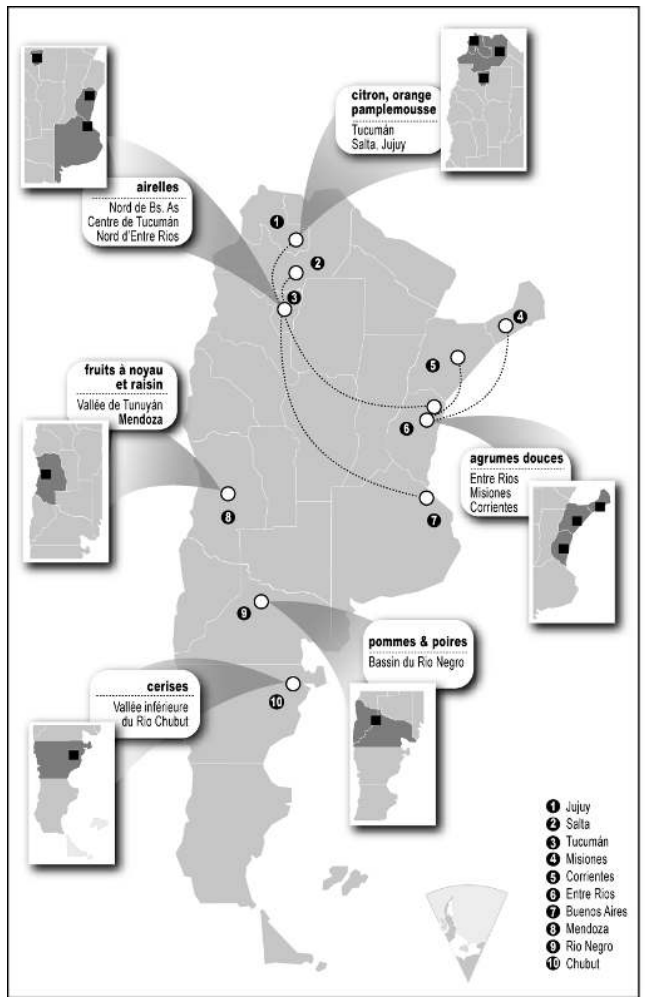

5 Les fruits à pépins occupent 57700 hectares (dont $65 \%$ correspondent aux pommes et $35 \%$ aux poires). L'Argentine se place au deuxième rang pour la production de pommes dans l'hémisphère sud, tandis qu'elle prend la première place pour la production de poires (FAO, 2004). La production nationale a atteint 1702000 tonnes en 2006.

6 Les agrumes occupent une superficie de 140432 ha dont $30 \%$ correspondent aux citrons, $60 \%$ aux agrumes de table (mandarines et oranges) et le reste aux autres espèces. Le volume total de production en 2006 a été de 3134382 tonnes tandis que les exportations représentent $44 \%$ du total des fruits frais expédiées à l'étranger, dont l'Union Européenne et la Russie sont les principaux marchés consommateurs.

7 À la fin des années 1990, la production de fruits rouges (airelles, cerises, fraises) a été encouragée pour l'exportation. Son expansion est liée aux stratégies des entreprises visant la qualité et la sélection productive pour s'insérer dans les niches de marché. Les fruits rouges représentent un des produits les plus dynamiques du commerce alimentaire international de la dernière décennie avec une croissance des exportations et de la valeur de la production (Obschatko, 2003). 
Tableau 1 : Les régions de production des fruits d'exportation Mains regions producing fruits for export

\begin{tabular}{|l|l|}
\hline \multicolumn{1}{|c|}{ Types de fruits } & \multicolumn{1}{c|}{ Régions de production } \\
\hline $\begin{array}{l}\text { AGRUMES } \\
\text { Citrons, oranges, pamplemousses } \\
\text { Oranges et mandarines (notamment) }\end{array}$ & $\begin{array}{l}\text { Nord-ouest : provinces de Jujuy, Salta et Tucumán } \\
\text { Mesopotamia : provinces de Misiones, Corrientes et Entre Ríos }\end{array}$ \\
\hline $\begin{array}{l}\text { FRUITS À PÉPINS } \\
\text { pommes et poires }\end{array}$ & $\begin{array}{l}\text { Patagonie : provinces de Río Negro et Neuquén (Oasis d'arrosage : Haute val- } \\
\text { lée de Río Negro, vallée moyenne et inférieure du fleuve Neuquén) }\end{array}$ \\
\hline $\begin{array}{l}\text { FRUITS À NOYAU } \\
\text { Pêches, prunes } \\
\text { RAISIN DE TABLE }\end{array}$ & Cuyo : province de Mendoza \\
\hline $\begin{array}{l}\text { FRUITS ROUGES } \\
\text { Airelles } \\
\text { Cerises }\end{array}$ & $\begin{array}{l}\text { Provinces de Buenos Aires, Tucumán et Entre Ríos } \\
\text { Patagonie : province de Chubut (Oasis d'arrosage : vallée inférieure du fleuve } \\
\text { Chubut) }\end{array}$ \\
\hline
\end{tabular}

8 Le tableau 2 montre la croissance du volume de fruits frais exportés et la valeur de la production obtenue les dernières années. Les fruits à pépins (pommes et poires) représentent $50 \%$ des exportations de fruits frais argentins tandis que $44 \%$ correspondent aux agrumes. Les exportations d'airelles et de fraises ont dépassé 16000 tonnes en novembre 2007 pour une valeur de 70000000 \$ US (22\% de plus qu'en 2006). L'Argentine est le deuxième pays exportateur d'airelles de l'hémisphère sud, tout juste après le Chili qui est un concurrent puissant en qualité et prix. Le principal marché extérieur sont les États-Unis (SAGPyA, Airelle - Rapport de conjoncture, décembre 2006). La commercialisation de la production de fruits frais est dirigée par les entreprises internationales et nationales, dont environ une vingtaine contrôlent 75 \% des ventes à l'étranger (Bendini et Steimbreger, 2005).

Tableau 2 : Le volume de production exporté et la valeur de la production de fruits (Source : SENASA, Bureau de Statistiques du Commerce Extérieur, Décembre 2007) Volume of exported production and value of the fruit production

\begin{tabular}{|c|c|c|}
\hline Année & Volume de production (tn) & Valeur de la production (Millons us\$) (en \$ courants) \\
\hline 2005 & 1456644 & 742863 \\
\hline 2006 & 1390328 & 766286 \\
\hline 2007 & 1563855 & 935731 \\
\hline
\end{tabular}

\section{La production des agrumes doux}

9 La région de la Mesopotamia (fig. 1 et 2) se situe au nord-est du pays. Il s'agit d'une zone dédiée notamment à la production d'agrumes doux (mandarines et oranges) ${ }^{3}$, suivis des citrons et des pamplemousses. C'est au cours des années 1980 que la production commence à se spécialiser en agrumes de table; un processus qui s'approfondit lors de la décennie passée, tandis que la province de Tucumán privilégie le modèle productif fondé sur la production des citrons. La production des agrumes doux est la plus importante au nord-est de la province d'Entre Ríos (départaments de Concordia et Federación), tant en superficie plantée (58 \% des surfaces de la Mesopotamia), qu'en production ( $71 \%$ du total de la Mesopotamia) selon la Fédération Nationale du Citrus - Federcitrus en 2006. La production de citrons et de pamplemousses diminue progressivement. Les petits producteurs dont le niveau de capitalisation est faible, maintiennent ces productions dans un système traditionnel et dirigent leurs ventes vers le marché national. L'activité agrumicole est intensive dans la 
gestion des plantations, elle exige une main-d'œuvre importante pendant la récolte et pour le conditionnement et l'emballage de la production.

\section{0-1980 : La mise en place de la filière agrumicole de l'Entre Ríos}

10 L'activité agrumicole s'est intallée à Concordia et à Federación pendant les années 1930. Pourtant la production est restée stable, limitée par les fléaux climatiques, les invasions et les épidémies, jusqu'au début des années 1960 au moment où la Commission provinciale des agrumes et la participation de l'Institut National de Technologie Agricole (INTA) pour les activités de recherche ont encouragé la production. La croissance de la superficie plantée a été accompagnée de l'installation de grandes entreprises, de capitaux régionaux qui cherchaient à produire, industrialiser et commercialiser des agrumes avec le soutien du gouvernement qui promouvait une politique de crédits accesibles, de préfinancement et de subventions pour l'exportation de jus d'agrume. Tandis que le marché national se consolidait, des conditions favorables pour l'exportation de fruits frais s'installaient. L'incorporation de technologies au niveau de la production agricole a entraîné l'introduction de nouvelles variétés de fruits; ceci a été accompagné de concert par le savoir technique et de nouvelles équipes pour l'industrie qui ont donné naissance à une véritable filière agricole industrielle avec divers niveaux d'intégration basés sur la production d'agrumes frais, tandis que le développement de la phase industrielle proprement dite (élaboration de jus et sous-produits) a été plus lent. La croissance des activités connexes (les industries du bois pour la fabrication d'emballages, les industries d'engrais, les entreprises d'équipement pour l'agriculture et d'emballage), l'équipement comercial et de services fonctionnels à la filière a contribué à la formation d'une économie régionale, favorisant la création de nouveaux emplois directs et indirects pour une grande partie de la population. La ville de Concordia est le centre de l'activité, tandis qu'à Chajarí (Federación), même s'il s'agit d'une petite ville, les activités sont d'un dynamisme croissant.

11 En 1971, la diminution de l'offre des agrumes sur le marché mondial ${ }^{4}$ a permis les premières exportations. Pindapoy $\mathrm{SA}^{5}$ a été remarquée alors comme une entreprise leader de cette activité dans le pays, organisant l'intégration verticale de la chaîne productive (production et emballage de fruits frais, de jus et de sous-produits) et l'expansion territoriale de la superficie des agrumes, des entreprises d'emballage et des industries de jus à Concordia, en Corrientes et en Misiones (fig. 2). 
Figure 2 : La culture d'agrumes dans le nord-est de la province d'Entre Rios Location of the citrus fruits production's area in Entre Rios

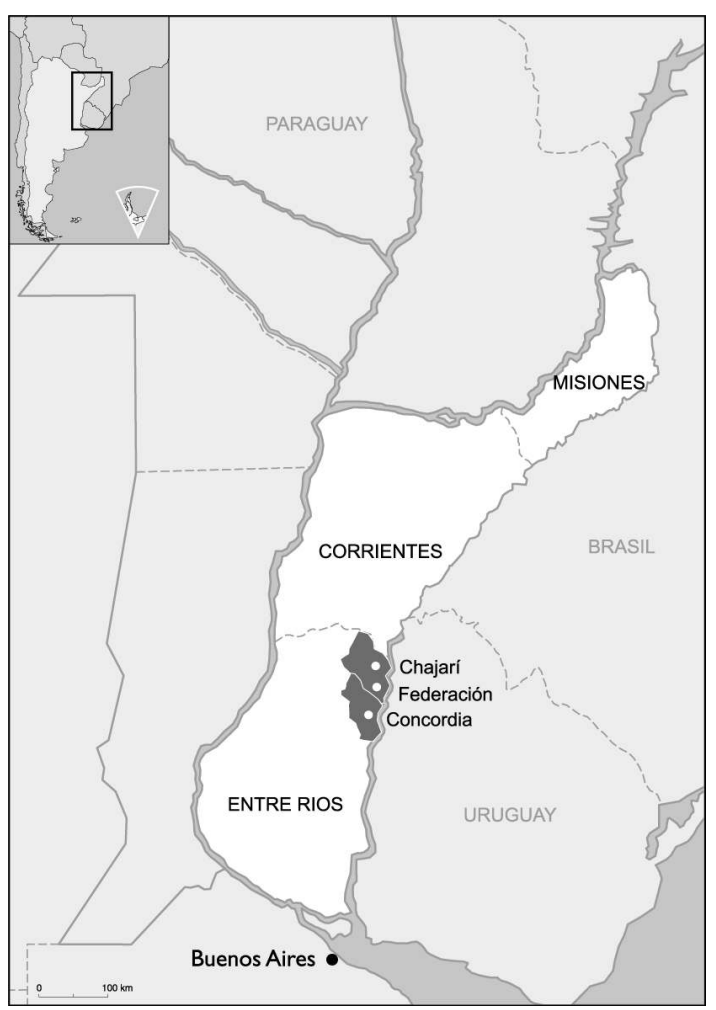

12 Au milieu des années 1970, la bactérie du chancre (Xanthomonas campestris) a menacé l'avenir de la production des agrumes. L'application d'un paquet technologique par l'INTA (Institut National d'Agronomie argentin) a contribué à l'amélioration du problème phytosanitaire. Au cours des années 1980, l'activité connaît une importante expansion par l'augmentation de la superficie plantée, la croissance de la densité de plants par hectare, l'amélioration des espèces, des rendements et de la production. Ce processus s'est accompagné de l'essor des exportations vers les pays de l'Europe et le Canada. Tout au long de cette période, la production de fruits frais soutient la croissance l'ensemble de la sphère agricole, tandis que l'industrie de jus et de sousproduits constitue une activité résiduelle qui dépend de l'oscillation des prix destinée au marché national.

\section{Les années 1990 : un contexte de crise et de restructuration productive}

En 1991, les changements de l'économie nationale provoquent le ralentissement de l'activité agrumicole jusqu'à déchaîner une crise en 1995, approfondie lors des années suivantes, accompagnée de conflits, de tensions et de négociations au fur et à mesure que de nouvelles formes d'intégration et d'articulation agro-industrielle apparaissaient. La non-intervention de l'État pendant ce processus, a agit comme un facteur favorable à la marginalisation des acteurs les plus faibles (vente d'exploitations agrumicoles et d'industries d'emballage endettées). Cette situation a favorisé la concentration des grandes entreprises qui cherchaient à augmenter l'intégration entre la production, l'emballage et la commercialisation. 

les activités de la filière, entraînant la stagnation de certains maillons de la chaîne de production et le déclin d'autres. Dans le scénario international, le processus de mondialisation en vigueur provoque la disparition des barrières commerciales des pays aux économies émergentes. En outre, les subventions agricoles à la production, les mesures de protection instaurées par les pays centraux et la superproduction agricole des pays producteurs concurrencent les productions agricoles, tout en plaçant l'Argentine dans une situation défavorable en tant que pays exportateur. De plus, plusieurs crises financières internationales (celle du Mexique en 1995, de l'Asie en 1997, du Brésil en 1998-1999) ont entraîné des processus récessifs pour l'économie argentine en provoquant la fuite de capitaux, des taux d'intérêt élevés et une diminution de la compétitivité des exportations argentines par la dépréciation de la monnaie des pays en crise. Ces phénomènes, qui ont touché tout le système agro-alimentaire argentin, se sont répercutés sur certaines particularités de la filière agrumicole d'Entre Ríos. À partir de 1995, les prix des agrumes sont inférieurs aux coûts de production moyens ; seuls les producteurs qui arrivaient à augmenter les rendements et la qualité de la production pouvaient faire face et s'adapter au nouveau contexte (INTA-Concordia, 2000). En 1998, la superficie des agrumes occupait $41500 \mathrm{ha}^{6}$; chiffre maintenu jusqu'à la fin de cette décennie. Le volume de production a enregistré des variations entre 1995 et 2000 (graphique 1) et une détérioration de la qualité des fruits du fait du moindre soin apporté aux plantations, des problèmes financiers et la faillite de certaines exploitations.

Les difficultés financières ont provoqué la décapitalisation de nombreux producteurs agrumicoles : les taux d'intérêt élevés et le manque de rentabilité n'ont pas permis un bon traitement des plantations. Les impôts et les augmentations de certaines taxes (péages, frets, etc.) ont pesé sur les coûts de production des entreprises de toute la chaîne productive au moment même où le prix des fruits augmentait au niveau du marché intérieur. Cette situation exposée aux asymétries d'échanges avec les autres pays producteurs sur le marché international des agrumes (l'Afrique du Sud, l'Uruguay, le Brésil), a provoqué la chute du volume et de la valeur des exportations. Ainsi, en 1999 et 2000, on observe une chute importante du volume exporté (fig. 3). 
Figure 3 : Évolution de la production des agrumes et des exportations entre 1996 et 2004 (Sources : FEDERCITRUS-INTA Concordia-SAGPyA-SENASA)

Evolution of citrus fruits production and export, 1996-2004

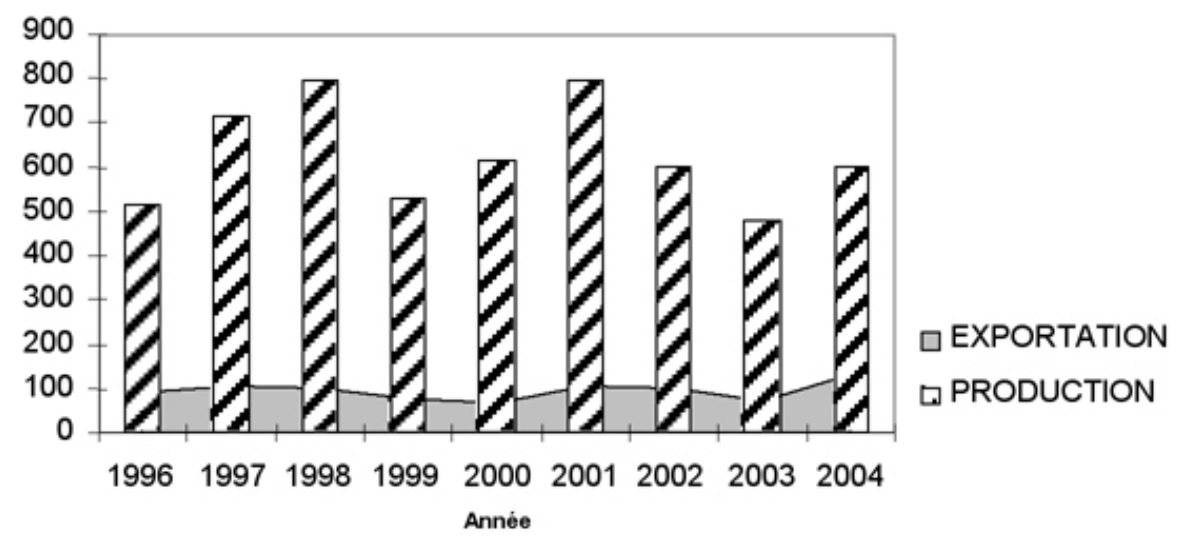

Source: Élabotion propre sur la base de FEDERCITRUS-INTA Concordia-SAGPyA-SENASA

\section{La restructuration productive de la filière agrumicole : le choix de l'exportation}

En 2002, l'activité agrumicole subissait une crise profonde. Dans ce cadre national très difficile (dévaluation de la monnaie, le non-paiement interne et externe), la situation économique de la province d'Entre Ríos s'est aggravée. Pourtant, la dévaluation de la monnaie a rendu possible les espoirs du secteur exportateur, malgré l'application de taxes à l'exportation, la crise de confiance nationale et l'insolvabilité de l'État argentin. Ainsi, les entreprises ont approfondi leur restructuration productive initiée à la fin de la décennie précédente, afin de se réadapter aux restrictions du contexte national et à la conjoncture internationale, en concentrant leurs efforts sur la stratégie de l'exportation. Les gros producteurs agrumicoles modernisés, les entreprises intégrées et les grandes entreprises agro-commerciales ont procédé à des modifications et des réajustements de l'ensemble de la filière productive, afin d'augmenter le volume de la production destiné au marché extérieur, en particulier, des pays exigeants en qualité et sécurité sanitaire. Des nouvelles technologies (augmentation de la densité de plants par ha ; sélection des variétés, pépinières propres, bourgeons certifiés, irrigation par goutte à goutte) ont été introduites dans la phase agricole. Le maillon d'emballage a diffusé l'utilisation de chambres froides à atmosphère contrôlée; certaines phases du processus d'emballage ont été automatisées; les entreprises se sont intéressées à la coordination des voies pour la commercialisation afin de contrôler la valeur de la production. Entre 2004 et 2006, le volume des exportations a augmenté de $42 \%$ (INTAConcordia). Bien que la Russie et l'Union Européenne demeurent les principaux acheteurs, d'autres destinations apparaissent (l'Europe de l'Est, le Canada, les pays de l'Asie). Actuellement, les expéditions vers l'étranger débutent en février ou mars (les variétés primeurs de mandarines) et durent jusqu'au mois d'octobre avec les envois d'oranges vers la Russie. Cette situation a contribué à dynamiser l'activité tout en provoquant l'élargissement de la saisonnalitéde la production d'agrumes de table pour l'exportation, qui était antérieurement située entre mai et septembre. 
17 Le maillon de l'emballage-exportation représente de nos jours l'axe de la croissance de l'industrie des agrumes de table. Parmi les 6 entreprises intégrées verticalement; une d'elles exporte $100 \%$ des fruits tandis que les autres 5 exportent entre 50 et $85 \%$ du volume emballé. Le reste de leur production est vendue sur le marché national. Ces 6 entreprises emploient environ $80 \%$ du total des ouvriers (phase agricole et d'emballage) en collaboration avec des producteurs capitalisés qui certifient la qualité des produits à exporter ${ }^{7}$ (information obtenue d'après les enquêtes directes réalisées aux entreprises d'emballage en 2006).

\section{Les acteurs agro-industriels actuels}

18 La restructuration de la filière depuis les années 1990 signale un changement qualitatif pour le développement de l'activité, le positionnement des acteurs, les stratégies des entreprises et les rapports sociaux de production.

\section{Le maillon agricole}

19 Aujourd'hui, on distingue au niveau du maillon agricole, trois types de producteurs.

20 - Les grands producteurs indépendants sont identifiables par l'incorporation de nouvelles techniques (densité de plants par ha, changement de variétés, voiles protecteurs, irrigation goutte à goutte, strict contrôle phytosanitaire) ; par l'obtention de meilleurs rendements et d'une plus grande qualité ; par une production adaptée au marché extérieur (traçabilité et label de qualité); par la vente d'un pourcentage important de leur production aux entreprises d'emballage-exportation nationales.

21 - Les producteurs " intégrés » aux entreprises d'emballage-exportation se caractérisent par la production de fruits de qualité sous la surveillance, le suivi de cultures, l'organisation $\mathrm{du}$ travail, l'assistance technique des entreprises d'emballage avec lesquelles ils sont sous contrat. Ce groupe connaît une lente perte d'autonomie.

22 - Les petits producteurs indépendants sont des exploitations de type familial, fondé sur un système d'exploitation traditionnel. Leur production est de qualité variable et destinée au marché national ou à l'industrie. On observe une tendance vers la diversification de la production ou la disparition de l'activité agricole.

\section{Le maillon commercial}

23 Au niveau du maillon commercial (emballage-exportation) se trouvent les acteurs principaux de la filière : les grandes industries d'emballage pour l'exportation et la commercialisation intégrées dans un processus de croissante concentration. Ce secteur comprend 3 types d'entreprises.

24 Les entreprises verticalement intégrées ont leur origine dans les producteurs agrumicoles locaux ou dans les industries d'emballage-commercialisation de la région. Dans les deux cas, les producteurs ont cherché la verticalisation afin d'augmenter l'échelle de production et de contrôler tout le processus (matière première, emballage, commercialisation). Les entreprises se sont integrées par l'achat de terres pour les plantations et les pépinières, bien qu'elles continuent également à acheter des fruits à des tiers, souvent de petits producteurs. Elles traitent environ $80 \%$ de la matière 
première et exportent entre 50 et $100 \%$ de leur production (enquête directe aux entreprises d'emballage, 2006). Elles utilisent des marques propres pour l'exportation et d'autres marques pour le marché national. La production qui ne possède pas les conditions minimales pour la commercialisation est destinée à l'industrie (jus et huiles essentielles). Ces entreprises essaient de coordonner leurs modes de commercialisation en formant des oligopsones. Elles ont recours à l'externalisation des services et à la flexibilité du travail dans l'objectif d'optimiser leur concurrence au commerce international et d'augmenter leur productivité. Des technologies de pointe sont combinées avec des formes d'organisation du travail modernes et traditionnelles. Le leadership et les avantages concurrentiels sont accompagnés d'une articulation avec les institutions officielles dédiées à la recherche, même si ces entreprises manquent de laboratoires propres pour développer la recherche.

Le consortium de producteurs se compose des petits et moyens producteurs agrumicoles grouper afin de commercialiser de plus gros volumes, réduire les coûts fixes pour accéder au marché étranger et s'adapter rapidement aux changements de la demande internationale, voire se fournir en matière première. Ils exportent la totalité de leur production. En outre, il dispose d'une industrie d'emballage et des équipements frigorifiques. Plus rarement, il traite des fruits pour le maché national.

Les entreprises agro-commerciales exportatrices forment un groupe hétérogène. De grandes entreprises non-locales qui dirigent le marché de fruits frais d'exportation argentin (Expofrut SA, Citrícola San Miguel SA et, plus récemment Ledesma SA), se sont insérées dans le maillon commercialisation-distribution de l'agro-industrie des agrumes d'Entre Ríos par l'achat de gros volumes d'agrumes doux pour le marché étranger. Elles achètent les fruits directement dans les exploitations et sous-traitent le service d'emballage auprès des industries locales, ou bien elles achètent les fruits déjà emballés. Ces entreprises commercialisent sous leurs propres marques. Après 1995, malgré la crise de la convertibilité dont la stagnation des ventes étrangères et la détérioration des conditions des échanges, deux de ces entreprises non locales ont poursuivi leur expansion, en conservant leur siège central, l'une dans le bassin du Río Negro (Expofrut SA), et l'autre dans la région du nord-ouest (San Miguel SA).

Certaines entreprises non locales, comme «Ingenio y Refinería El Tabacal », qui ont des activités agrumicoles dans la région du Nord-Ouest argentin, se sont incorporées au maillon commercialisation-exportation de la filière d'Entre Ríos ces dernières années. Elles sont les donneurs d'ordre d'entreprises d'emballage sous-traitantes et traitent les fruits achetés à des tiers.

Enfin, une entreprise locale qui a commencé son activité en tant que fournisseurs de service (frigorifiques, déverdissage, drencher ${ }^{8}$ ) pour la chaîne de production d'agrumes, a créé une entreprise d'emballage en 2003, comme une autre phase de prestation de services à des tiers pour emballer des agrumes d'exportation. Aujourd'hui, cette société emballe une petite partie de sa propre production.

29 Le processus de commercialisation pour l'exportation est devenu de plus en plus complexe lors des dernières années. En effet, les grandes entreprises d'emballage locales prennent l'habitude de s'organiser entre elles afin de concrétiser certaines ventes. Il s'agit d'une stratégie qui favorise un volume plus important de certaines variétés et, en outre, qui accélère le travail en juste à temps des entreprises d'emballage. En plus, une des plus grandes entreprises d'emballage d'exportation (Fama SA) s'est associée à San Miguel SA en 2005 pour exporter des agrumes doux et des 
citrons. Autrement dit, les articulations entre les entreprises d'emballage exportatrices locales et celles non-locales, qui opèrent dans la même filière agrumicole, sont conformes à la logique entrepreneuriale qui consiste à augmenter l'échelle et la rentabilité dans un marché mondialisé. Dans le cadre actuel, les rapports des entreprises comprennent une trame complexe et flexible traduite dans les associations et les alliances faites et défaites selon les besoins conjoncturels.

\section{Conclusion} mondiale ouvre une voie favorable à l'exportation de fruits frais argentins de contresaison. Ce phénomène provoque une croissance de la production et des changements dans les formes de commercialisation. De plus, l'ensemble des activités liées au processus de production et de traitement postérieur à la récolte (classification, emballage, refroidissement, contrôle de qualité, etc.) se dynamise, ce qui ajoute de la valeur au produit et lui donne des caractéristiques propres au processus agroindustriel.

31 Dans cet article, nous avons analysé les traits principaux du profil productif de la filière agro-industrielle des agrumes d'Entre Ríos : sa croissance à partir des années 1960, sa stagnation et la crise au milieu de la décennie passée qui a entraîné un processus de restructuration productive dans le contexte de changements des économies nationale et internationale. En 2002, la forte dévaluation de la monnaie a été mise à profit par la filière pour développer l'exportation des agrumes de table de contre-saison, avec le rôle moteur du secteur de l'emballage-exportation à la tête de la croissance de la filière. Les nouvelles dynamiques de ce secteur s'expriment à travers les intégrations et les articulations entrepreneuriales étroites et formelles avec les entités publiques et privées (INTA, laboratoires de contrôle de qualité) pour générer des synergies permettant de répondre correctement et concurrentiellement au marché étranger, consolidant ainsi leur croissance.

Les rapports entre la phase agricole et la phase commerciale de la filière agrumicole, se sont intensifiés à travers la coordination et les contrôles techniques plus étroits et une intervention plus importante des entreprises de commercialisation (emballageexportation) dans les processus productifs de matière première. La structure agricole locale acquiert une nouvelle forme moyennant un processus qui, d'un côté, augmente la dépendance des producteurs vis-à-vis des décisions des entreprises et qui, d'un autre côté, provoque de la résistance et des conflits avec les agents les plus vulnérables (petits producteurs). Les grandes entreprises d'emballage déploient des stratégies pour exercer un contrôle plus contraignant sur le travail (ajustements concernant la quantité et la qualité du travail, le lien contractuel précaire, bas salaires) sous la protection de la législation du travail en vigueur qui favorise la fléxibilité de travail. Le contrôle de qualité est un point de tension, de même que la question de la sécurité sanitaire, puisque les entreprises doivent garantir la traçabilitédu produit pour le marché de destination. Les exigences se manifestent aussi sur la pression des prix, la fréquence d'envois, les normes que doivent remplir les bâtiments, les normes d'hygiène et de salubrité qui doivent suivre les ouvriers, tant au niveau de la production agricole qu'au niveau des entreprises d'emballage. Pourtant, les entrepreneurs de grandes sociétés d'emballage ont pu augmenter les envois à l'étranger et améliorer leur 
rentabilité, de même que les grands producteurs agrumicoles. Par contre, les petits producteurs et les entrepreneurs du secteur de l'emballage qui n'accèdent pas au marché extérieur sont dans une situation de marginalisation, de précarité, qui les mène vers l'exclusion de l'activité. En conséquence, l'actuel dynamisme de la filière vis-à-vis de la demande extérieure des agrumes frais bénéficie de façon inégale aux acteurs de la filière.

\section{BIBLIOGRAPHIE}

BENDINI (M.), STEIMBREGER (N.), 2005. - « Integración agroalimentaria. Trayectorias empresariales comparadas en la fruticultura argentina de exportación », Dans CAVALCANTI (S.), NEIMAN (G.), Acerca de la Globalización en la Agricultura. Territorios, empresas y Desarrollo local en América Latina, Buenos Aires, Ediciones Ciccus, p. 25-37.

LAMANTHE (A.), 2001. - «Petites entreprises à l'épreuve de la rationalisation. Le cas de la transformation des fruits et légumes en Provence, des années soixante aux années quatre-vingtdix », Revue Française de Sociologie, $\mathrm{n}^{\circ}$ 42-3, p. 509-536.

MERCIER (D.), TANGUY (C.), 2004. - « Les cas des oranges dans la région Cuenca del Plata, zone frontière entre l'Argentine et l'Uruguay ", Colloque International Réseau CDP - Cuenca del Plata/ IPEALT, Université de Toulouse Le Mirail (France) 1-3 juillet 2004, Version CD-rom, p. 1-18.

овSCнатко (Е.), 2003. - « Nuevos Productos agropecuarios de alto valor en la Argentina. Panorama y aspectos estratégicos », Revista Argentina de Economía Agraria, Mendoza, Argentina, p. 73-88.

RECA (L.), PARELLADA (G.), 2001. - El sector agropecuario argentin, Editorial Facultad de Agronomía, Universidad de Buenos Aires, 120 p.

ROFMAN (A.), VON STORCH (V.), GUTMAN (V.), 2003. - "Situación contemporánea de las economías regionales. Estrategias para incorporar a un modelo de desarrollo con equidad social", Noticias del Centro Humboldt, Buenos Aires, 5 avril 2003, p. 1-30.

TADEO (N.), PALACIOS (P.), TORRES (F.), 2006. - Agroindustria y Empleo. Complejo agroindustrial citrícola del noreste entrerriano, Buenos Aires, Editorial La Colmena, 215 p.

\section{NOTES}

1. En 1991, le gouvernement installe le régime de convertibilité en Argentine fixe la parité d'échange qui établit, par loi du Congrès national, l'égalité entre le peso argentin et le dollar américain dans le but de mettre fin aux crises inflationnistes et aux demandes des créanciers de dettes. Ce régime a entraîné une valorisation financière, l'endettement et le transfert de ressources à l'extérieur. Le recul de ce type d'échange a eu incidence sur les exportations par une perte importante de concurrence des productions. Parallèlement, il se produit une remarquable restructuration des activités économiques, une nouvelle organisation de la structure sociale (croissance du chômage et du sous-emploi, affaiblissement de la trame sociale) aussi bien dans le domaine urbain que dans le domaine rural. 
2. La postconvertibilité : le peso argentin a subit une remarquable dévaluation. Trois caractéristiques mettent l'accent sur les économies régionales basées sur la production agricole. 1- les changements des prix relatifs qui n'ont pas favorisé tous les acteurs mais à ceux qui étaient concentrés et dans une situation avantageuse afin de faire face au changement. 2- disparition du crédit et des sources de financement, situation aggravée par cessation de paiement et dévaluation. 3- détérioration du salaire réel dû à l'impact de l'inflation provoquée par la dévaluation (Rofman et al., 2003).

3. La dénomination des agrumes doux pour les oranges et les mandarines répond à une distinction spatiale et à la spécialisation productive faite pour le citron, localisé en particulier en Tucumán, région du nord-ouest (fig. 1).

4. La production des pays méditerrannées a été touchée par des aléas climatiques.

5. Il faut noter que Pindapoy SA a été une entreprise nationale familiale, pendant les soixante ans de son existence (Tadeo, Palacios, Torres, 2006).

6. En 1995, la superficie plantée d'agrumes était de 46778 hectares (Recensement provincial agrumicole - Entre Ríos, 1995).

7. Depuis 2004, les fruits destinés à l'UE doivent être certifiés conforme la norme EUREP-GAP, GLOBAL-GAP depuis 2007 [www.globalgap.org].

8. Déverdissage : technique qui consiste à conserver les mandarines primeurs dans des chambres froides pour que leur peau soient plus colorées. Drencher : traitement fongicide préventif pour les agrumes qui sont conservés temporairement ou bien qui sont exportés.

\section{RÉSUMÉS}

Les changements produits dans la consommation, surtout dans les pays les plus développés mais, en particulier, dans certains secteurs de la population des pays en voie de développement, ont augmenté la demande de produits frais. En Argentine, l'arboriculture fruitière connaît un fort essor depuis 1990, par sa place stratégique sur le marché de fruits frais de contre-saison pour l'hémisphère nord. Cet article analyse le cas de la filière des agrumes de table d'Entre Ríos et le processus de restructuration productive qui l'anime à partir des années 1990, comme réponse aux changements du cadre économique national et international et aux exigences, tant en qualité qu'en termes de sécurité alimentaire, du marché étranger des fruits frais.

Changes in the consumption, especially in most developed countries but also in certain sectors of the population in developing countries, have increased the demand of fresh products. Since the beginning of the 1990 fruit farming in Argentina has been increasingly develloping because of its strategic position for off-season fresh fruits offer in the Northern Hemisphere. This paper analyzes the case of the citrus agro-industrial complex in Entre Ríos and its productive restructuring process. The changes in the national and international context and the market demands for high quality fresh fruits has induced new requirements for presentation health and safety. This paper presents an empirical and analytical approach based on quantitative and qualitative information.

Los cambios en el consumo, sobre todo en los países más desarrollados, pero también en determinados sectores de población de los países en desarrollo, han aumentado la demanda de productos frescos. La fruticultura en la Argentina es una de las producciones con creciente 
dinamismo desde 1990 por su posición estratégica en la oferta de frutas frescas de contraestación en el Hemisferio Norte. Este artículo analiza el caso del complejo agroindustrial citrícola de Entre Ríos y su proceso de reestructuración productiva a partir de la década pasada, como respuesta a los cambios que se manifestaron en el contexto económico nacional e internacional y las exigencias de un mercado externo de frutas frescas de mayor calidad, presentación y seguridad sanitaria. Un enfoque empírico y analítico facilita la utilización de información cuanticualitativa.

\section{INDEX}

Index géographique : Argentine, Entre Ríos

Palabras claves : cítricos dulces, complejo agroindustrial, fruta fresca, reestructuración productiva

Keywords : agro-industrial complex, productive restructuring

Mots-clés : filière agro-industrielle, restructuration productive

\section{AUTEUR}

\section{NIDIA TADEO}

Département de Géographie, Université Nationale de La Plata, Faculté des Humanités et Sciences de l'Éducation, Calle 48, entre 6 y 7, quinto piso, Oficina 506, La Plata - CP 1900 Buenos Aires, Argentine, nidiatadeo@yahoo.com.ar 